\title{
On the Structural Difference Between the Evolutionary Approach of Young and that of Kandori, Malaith, and Rob
}

\section{Hans Jørgen Jacobsen, Mogens Jensen, and Birgitte Sloth*}

\author{
June 1999
}

This version: 1. December 1999

\begin{abstract}
We provide robust examples of symmetric two-player coordination games in normal form that reveal that equilibrium selection by the evolutionary model of Young (1993) is essentially different from equilibrium selection by the evolutionary model of Kandori, Mailath and Rob (1993).
\end{abstract}

${ }^{*}$ Institute of Economics, University of Copenhagen, Studiestæde 6, DK-1455 Copenhagen K, DENMARK 


\section{Introduction}

The evolutionary models for games of Kandori, Mailath, and Rob (1993), henceforth KMR, and of Young (1993) have many similarities.

They are alike in concept: both study dynamic processes defined from some kind of boundedly rational behavior, where players try to learn from observations of the behavior of other players.

They are alike in formalization: both first define an "unperturbed" dynamic process where players play best replies to the behavior they observed, and then define a "perturbed" process by adding a small trembling probability to the unperturbed process.

They are alike in mathematics: for both models the unperturbed process is a Markov chain and the perturbed one (also a Markov chain) is a regular perturbation of this. The very strong equilibrium selection results are in both models obtained by studying the stochastically stable states for the perturbation. ${ }^{1}$

They are alike in results: both of the above mentioned papers study equilibrium selection in simple $2 \times 2$ coordination games and both find support for the "risk-dominance" selection criterion of Harsanyi and Selten (1988).

They are even alike in what drives the results: for both models the selection of the riskdominant equilibrium hinges on the fact that this equilibrium has, according to the unperturbed process, the largest basin of attraction, and the little trembling of the perturbed process implies (when there are only two strategies on each side), that this property is turned into the stronger one of being almost the only equilibrium observed.

These similarities may give the impression that the two alternative evolutionary approaches are "structurally" close and that they will quite generally give the same equilibrium selection. In that case it would be more or less a matter of taste or convenience which type of model should be preferred.

We demonstrate here, however, that the two models are structurally different and may well give rise to completely different equilibrium selections. We give two examples of games which are very close to the games studied in the mentioned contributions of KMR and Young. Both are simple and symmetric two-player coordination games, each with two strict Nash equilibria. The games also have some non-equilibrium strategies (the strategies

\footnotetext{
${ }^{1}$ For formal definitions of Markov chains and associated concepts such as absorbing sets, regular perturbations, and stochastically stable states, and for the graphwise characterization of the latter used below to identify long run behavior, see the orginal contributions by KMR (1993) and Young (1993).
} 
usually omitted "for simplicity"). The presence of the non-equilibrium strategies makes the KMR and the Young processes work differently and result in opposite equilibrium selection.

In the KMR approach, there is one population of $N$ individuals and in each round everybody is randomly matched to an opponent with whom he plays a symmetric, two-player game. After a round of play, it can be observed how the population played. According to the unperturbed KMR process there is for each player in each round independently a probability $\delta$ of "strategy revision": with probability $1-\delta$ the player takes the same action as in the last round, and with probability $\delta$ he takes an action that is a best reply to the mixed strategy made up of the relative frequencies of strategies in last round's play by the population. ${ }^{2}$ How the population played in rounds before the immediately preceding one is (forgotten and) not used. The perturbed process is defined by the modification that there is for each player in each round independently a probability $\varepsilon$ that the player takes a random action. The interest is in the perturbed process for small $\varepsilon$.

In the Young approach there is a population for each of the (two) player positions in the game. In each round of play, one player is picked randomly from each population and the picked players play the game between them. After the round everybody can observe what was played and everybody keeps for each position in the game a record of the actions taken the last $m$ times the game was played. According to the unperturbed Young process, a player, who has been picked to play, draws a random sample of $k$ observations from the record on the opponent, $k \leq m$, and plays a best reply to the mixed strategy made up of the relative frequencies of strategies in the sample. The perturbed process is defined by assuming that in each round and for each picked player independently there is a small probability $\varepsilon$ that the player takes a random action. Again, the interest is in the perturbed process for small $\varepsilon$.

There are several differences between the two approaches, some of which are inessential (for equilibrium selection) and some of which are arbitrary. The strategy revision probability $\delta$ appearing in the KMR, but not in the Young, approach is inessential; the KMR process will give the same equilibrium selection for all $\delta \in] 0,1]$.

The difference with respect to the number of populations is arbitrary: a KMR process could

\footnotetext{
${ }^{2}$ Or perhaps he takes an action that was a most or more successful one in last round's distribution of play. Since the KMR paper only considers games with two strategies for each player, there is no sharp distinction between the best reply dynamics that we consider here, and imitation dynamics, and both are covered as alternative "Darwinian dynamics" by the original formulation. Since we study here a KMR process for games with more strategies, we have to make a choice. We follow such contributions as Nöldeke and Samuelson (1993), Kandori and Rob (1996), and Robles (1997), and study best reply dynamics.
} 
as well be defined with one population for each of the player positions in the game, and for symmetric games a Young process could as well be defined with just one population. The number of populations may, however, matter for equilibrium selection, a feature well-known from, e.g., replicator dynamics. To demonstrate that it is not simply the difference in the number of populations that make the two approaches structurally different, we consider one-population versions of both processes, defined, of course, only for symmetric games. For the one-population Young process it is assumed that in each round one player is picked randomly and takes an action. He will receive payoff depending on his own action and the action taken in the next round as given by the considered game. He forms an expectation of next round's action as in the usual Young approach, by drawing a $k$-sample from the record of the actions taken in the last $m$ preceding rounds, etc. It will be clear from the forces driving our results, however, that one could also construct examples where both types of process were defined with two populations, or where one were defined with one and the other with two, and still get that equilibrium selection would be different between the two approaches.

The sampling occurring in the Young process when $k<m$ is a third difference. It is known that the presence and degree of sampling may indeed be of importance for equilibrium selection, but it is not essential to the fact that the two approaches give different equilibrium selection. In our first example, given in Section 2, we consider a Young process with full sampling, $k=m$, and the two processes select opposite equilibria. The basic effect that gives rise to the difference would disappear if, in the Young process, one assumed "sufficiently little sampling", as suggested by Young and often seen in applications. However, our second example, given in Section 3, demonstrates, that with less than full sampling, and in particular with sufficiently little sampling, another effect becomes important and may imply that the two approaches give opposite equilibrium selection again.

We are thus led to conclude that the difference in equilibrium selection in our examples must be explained by the remaining difference, i.e., by the fact that in the KMR approach players learn from observing the behavior of the full population in only the last round, whereas in the Young approach players learn from observing the behavior of players in a string of earlier matches going back in time. This means that there is no way around the difficult question of how players learn: is the KMR or the Young learning approach the most plausible? This question would not be important for the issue of equilibrium selection if the two processes gave rise to the same selection. ${ }^{3}$

\footnotetext{
${ }^{3}$ One can, of course, redefine the two approaches until they are the same. If one assumes in the KMR approach that players learn from population behavior in the last $m$ rounds of play, and in the Young approach that in each round all players are matched with an opponent, then the two processes become identical with respect to from what players learn. Our results should then rather be read that
} 
Each of the examples we give is robust; if the payoffs are changed a little, the two processes still select the two different equilibria as before. Further, the examples are constructed such that each player gets the same payoff in both of the game's strict equilibria, so by changing the payoffs a little in one direction or the other, one can make one equilibrium or the other Pareto dominating. Thus, an implication of our analysis is that it is not the case that one of the processes is systematically more reluctant to select Pareto inferior equilibria than the other. Rather, there are cases where the Young process selects a Pareto efficient, and the KMR process a Pareto inferior, equilibrium, and cases where it is the opposite.

\section{First Example}

Below a symmetric two-player normal form game with row player payoffs is displayed. It has two equilibria in pure strategies, $(a, a)$ and $(d, d)$. We will examine long-run behavior according to the two described processes for this game.

\begin{tabular}{|l||l|l|l|l|}
\hline & $a$ & $b$ & $c$ & $d$ \\
\hline \hline$a$ & 10 & 0 & 6 & 8 \\
\hline$b$ & 9 & 7 & 0 & 0 \\
\hline$c$ & 8 & 12 & 0 & 0 \\
\hline$d$ & 0 & 0 & 7 & 10 \\
\hline
\end{tabular}

In the KMR approach a state is a description of the population's play in the last round, a vector $\left\{\omega_{a}, \omega_{b}, \omega_{c}, \omega_{d}\right\}$, where $\omega_{a}$ is the number of players who used strategy $a$ etc., and $\omega_{a}+\omega_{b}+\omega_{c}+\omega_{d}=N$. The unperturbed and perturbed KMR processes are defined as described above. If a player who has been drawn for strategy revision has a tie - several actions are best replies against last round's population behavior - it is for the unperturbed process assumed that the player picks a random best reply, but such that all best replies have positive probability. Each process will for any two states give rise to a well-defined transition probability and hence each is a homogeneous Markov chain.

In the Young approach a state is a description of play in the last $m$ rounds. Where there is just one population, this means that a state is a vector $\left(\omega_{1}, \omega_{2}, \ldots, \omega_{m}\right)$, where $\omega_{i}$ is the strategy, $a, b, c$, or $d$, taken $i$ rounds before the current one. The unperturbed and perturbed Young processes are defined as described above. In this section we will assume

it is essential for the equilibrium selection obtained by any one of the approaches from what players are specifically assumed to learn, e.g., for the KMR approach if players are assumed to learn from only the last, or from the last $m$, rounds of population play. 
full sampling, $k=m$. If there is a tie, all best replies have positive probability. Again, each of the processes will give rise to well-defined transition probabilities and hence is a homogeneous Markov chain.

Both in the KMR and in the Young approach long run behavior is given by the stochastically stable states defined from the perturbed process. These are the only states that will be observed frequently in the long run when the trembling probability is small. Only states that belong to absorbing sets (recurrent classes) of the unperturbed process can be stochastically stable. It is therefore of interest first to find these absorbing sets, so first we show that only the states corresponding to the pure strategy equilibria are absorbing,

Proposition 2.1. The sets $\{(N, 0,0,0)\}$ and $\{(0,0,0, N)\}$ are the only absorbing sets for the unperturbed KMR process.

Proof: Due to the best reply structure of the considered game, if one of the above states has been reached the state in the next round will be the same for sure according to the unperturbed process. Any player drawn for strategy revision will have the action already used as best reply. Start in any other state. It has positive probability according to the unperturbed process that in all of the following three rounds all players are drawn for strategy revision. After one such round (with positive probability) everybody plays the same, and after two more either everybody plays $a$ or everybody plays $d$, and one of the two states has been reached.

Proposition 2.2. The sets $\{(a, \ldots, a)\}$ and $\{(d, \ldots, d)\}$ are the only absorbing sets for the unperturbed Young process.

Proof: Clearly, if one of the two states has been reached the unperturbed process stays in it for sure. Start in any other state. Now if $a$ is a best reply in the state, then with positive probability $a$ is played and the new state can only contain more $a$ 's. Then in the next round $a$ is still a best reply etc., and so the process will with positive probability reach the state $(a, \ldots, a)$ after at most $m$ rounds. Similarly, if $d$ is a best reply from the initial state, then $d$ remains a best reply and the process reaches $(d, \ldots, d)$ with positive probability. If neither $a$ nor $d$ is a best reply to the initial state, $b$ or $c$ or both must be. As long as this is the case the state will be filled up with $b$ 's and $c$ 's. This may imply that suddenly $a$ or $d$ is best reply and we are done by the above. If $a$ and $d$ continue not to be best reply, then after less than $m$ rounds $b$ will stop being best reply, since $b$ is not a best reply to $b$ or $c$ or to any combination of only $b$ s and $c$ s. The state will now be filled up with $c$ 's. This will 
eventually make $d$ a best reply and we are done. So, from any other state it has, according to the unperturbed process, positive probability to reach $(a, \ldots, a)$ or $(d, \ldots, d)$.

Now we characterize the stochastically stable states for each process. First, if players learn and tremble as described by the KMR process, and the trembling probability is small, then only the equilibrium strategy $d$ will be observed frequently,

Proposition 2.3. If $N$ is sufficiently large, then $(0,0,0, N)$ is the unique stochastically stable state for the KMR process.

Proof: Only $(N, 0,0,0)$ and $(0,0,0, N)$ are candidates for being stochastically stable. Let the state be $(0,0,0, N)$. If $\left[\frac{1}{6} N\right]_{+}$players simultaneously tremble and play $a$, then in the next round the only best reply to the state then prevailing is $a$. It then has positive probability according to the unperturbed process that all players are drawn for strategy revision, and the process ends up in the absorbing state $(N, 0,0,0)$, where all play $a$. Obviously, the described trembling is the one that requires the fewest trembles, so the resistance in the transition from state $(0,0,0, N)$ to state $(N, 0,0,0)$ is $\left[\frac{1}{6} N\right]_{+}$.

Now start in $(N, 0,0,0)$. With $\left[\frac{1}{8} N\right]_{+}$simultaneous trembles by the players to strategy $b$, the best reply to the current state will change to $b$. Now there is positive probability according to the unperturbed process that all players are drawn for strategy revision and hence choose $b$. Again in the next round it has positive probability that all players get a "learning draw" and then all will play c. Finally, in the next round it has positive probability that all players learn and all play $d$. So, the resistance in the transition from $(N, 0,0,0)$ to $(0,0,0, N)$ is $\left[\frac{1}{8} N\right]_{+}$. Since this is smaller than $\left[\frac{1}{6} N\right]_{+}$for $N$ sufficiently large, the stochastically stable state is $(0,0,0, N)$.

Second, if players learn and tremble as described by the Young process, and the trembling probability is small, then only the equilibrium strategy $a$ will be observed frequently,

Proposition 2.4. If $m$ is sufficiently large, then $(a, \ldots, a)$ is the unique stochastically stable state for the Young process.

Proof: Like for the KMR process, the minimal number of trembles required to go from $(d, \ldots, d)$ to $(a, \ldots, a)$ is $\left[\frac{1}{6} m\right]_{+}$, which is thus the resistance in this transition.

Now consider transition from $(a, \ldots, a)$ to the $(d, \ldots, d)$. This must start with a number of trembles to either $b, c$, or $d$ which are sufficient to make the best reply shift away from $a$. Consider first trembles to $c$. It will require $\left[\frac{10}{11} m\right]_{+}$such trembles before the best reply 


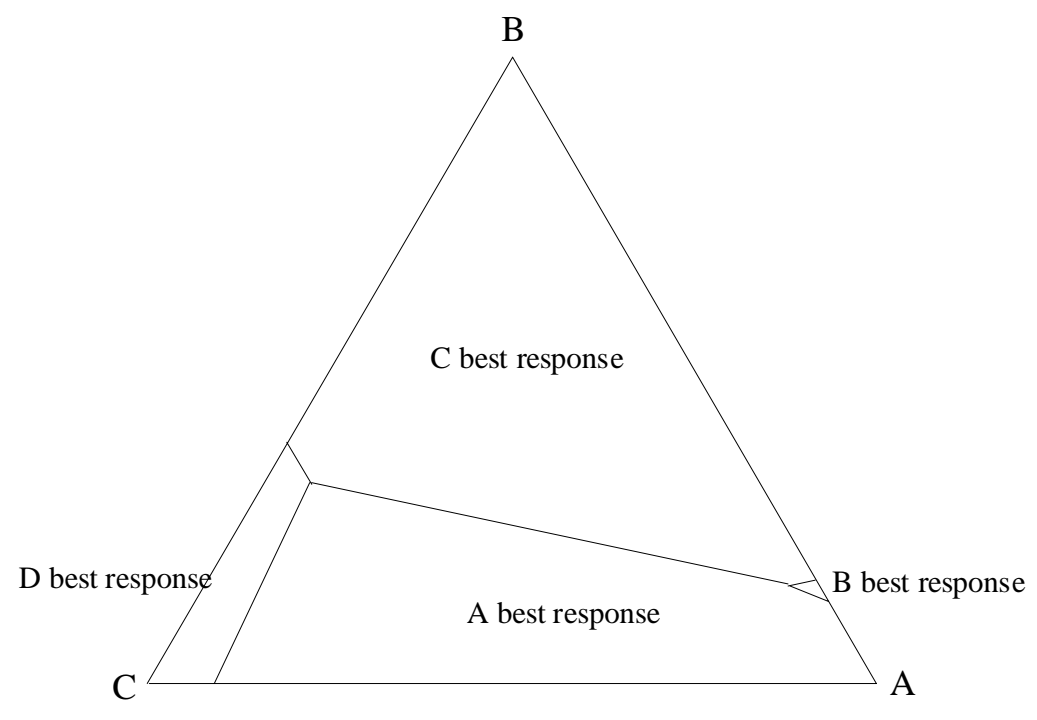

Figure 2.1: The best response regions given only actions $\mathrm{a}, \mathrm{b}$ and $\mathrm{c}$ are used by opponent.

shifts (to $d$ ). For trembles to $d$, it requires $\left[\frac{5}{6} m\right]_{+}$to make the best reply shift (again to $d)$. Finally, consider trembling to $b$. First it requires $\left[\frac{1}{8} m\right]_{+}$of these before the best reply changes and it changes to $b$. Without further trembling $b$ will be best reply and hence be played until the state consists of $\left[\frac{1}{6} m\right]_{+}$times $b$ (and $m-\left[\frac{1}{6} m\right]_{+}$times $a$ ). Then $c$ becomes best reply and remains so until the record consists of $\left[\frac{1}{6} m\right]_{+} b$ 's, $\left[\frac{1}{12} m\right]_{+} c$ 's (and $m-\left[\frac{1}{6} m\right]_{+}-\left[\frac{1}{12} m\right]_{+} a$ 's). But then $a$ is best reply again, and without further trembling the process returns to $(a, \ldots, a)$. So, the $\left[\frac{1}{8} m\right]_{+}$trembles are not sufficient to escape this state, further trembling is required.

So, go back and assume that just after the $\left[\frac{1}{8} m\right]_{+}$trembles to $b$ there is further trembling. If this is to $d$, it will require $\left[\frac{89}{144} m\right]_{+}$additional trembles to make the best reply shift (to $d$ ). If it is to $c$, it requires $\left[\frac{89}{132} m\right]_{+}$additional trembles to make the best reply shift (again to $d$ ). Finally, if it is to $b$ again, it requires further $\left[\frac{89}{582} m\right]_{+}$trembles to make the best reply shift (to $c$ ), in which case the unperturbed process indeed will go to $(d, \ldots, d)$ with positive probability. See figure 2.1, which shows the best replies given only the strategies $a, b$, and $c$ are used.

The total resistance in the transition from $(a, \ldots, a)$ to $(d, \ldots, d)$ is therefore $\left[\frac{1}{8} m\right]_{+}+\left[\frac{89}{582} m\right]_{+}$ which, for $m$ sufficiently large, is strictly higher than $\left[\frac{1}{6} m\right]_{+}$. So, the stochastically stable state for the Young process is $(a, \ldots, a)$. 
The obtained difference in selection mainly follows from a difference between the two approaches with respect to the number of players who can, with positive probability according to the unperturbed process, update behavior in a single round. In the KMR model it is possible that all players update behavior in one and the same round. Thus, it is possible that the behavior of the entire population shifts from one round to the next. This is different from the Young model, where exactly one player updates in each round and therefore the frequencies of play in the record only change slowly. Using a landscape metaphor, if the basin of attraction is a deep hole, then in the example it is possible for both processes to climb to the top of a platform standing in the middle of the hole by a little trembling, but to escape from the hole it is necessary to make a big jump to the edge of the hole. The KMR process is capable of doing that whereas the Young process can only make a small jump and will therefore fall down into the hole again.

\section{Second example}

Sufficiently little sampling in the Young process will imply that the two approaches select in the same way in the above example. Our second example reveals that also with partial, in particular with very little, sampling in the Young process this may well select differently than the KMR process, only due to another effect. We consider the symmetric two-player game with row player payoffs as given below. The game has two pure strategy equilibria, $(a, a)$ and $(e, e)$.

\begin{tabular}{|l||l|l|l|l|l|}
\hline & $a$ & $b$ & $c$ & $d$ & $e$ \\
\hline \hline$a$ & 10 & 6 & 9 & 8 & 2 \\
\hline$b$ & 0 & 0 & 5 & 0 & 6 \\
\hline$c$ & 8 & 9 & 0 & 0 & 0 \\
\hline$d$ & 7 & -3 & 10 & 0 & 0 \\
\hline$e$ & 0 & 8 & 0 & 7 & 10 \\
\hline
\end{tabular}

The processes are defined as above except for the modification that we now assume $k \leq m / 3$ in the Young process, and that states for both processes are redefined to capture that the game now has five strategies.

As for the first example only states corresponding to the two pure strategy equilibria can be absorbing. To demonstrate this goes just like the proofs of Propositions 2.1 and 2.2 above. We therefore state without proof, 
Proposition 3.1. The sets $\{(N, 0,0,0,0)\}$ and $\{(0,0,0,0, N)\}$ are the only absorbing sets for the unperturbed KMR process.

Proposition 3.2. The sets $\{(a, \ldots, a)\}$ and $\{(e, \ldots, e)\}$ are the only absorbing sets for the unperturbed Young process.

The following propositions state that the long run behaviors of the two processes are again different. The KMR process selects the equilibrium $(a, a)$, while the Young process selects $(e, e)$.

Proposition 3.3. If $N$ is sufficiently large, then $(N, 0,0,0,0)$ is the unique stochastically stable state for the KMR process.

Proof: To escape from the state $(0,0,0,0, N)$ at least $\left[\frac{4}{9} N\right]_{+}$trembles are required before the best reply changes to an action different from $e$. If the mistakes are all to $a$, then there is, according to the unperturbed process, positive probability of transition to the state $(N, 0,0,0,0)$. So, the resistance in this transition is $\left[\frac{4}{9} N\right]_{+}$.

From state $(N, 0,0,0,0)$, if trembling is to actions different from $b$, more than $\left[\frac{4}{9} N\right]_{+}$ trembles are required before the best reply changes. For trembles to $b$ only $\left[\frac{2}{5} N\right]_{+}$mistakes are required before the best reply changes to $c$. Note that $b$ can only be a best reply if the state contains more than $\frac{1}{2} N$ times $e$ (otherwise $a$ will be better).

For $e$ to be a best reply to a state with at most $\left[\frac{2}{5} N\right]_{+} b$ 's, and only $a$ 's and $c$ 's otherwise, further $\left[\frac{23}{93} N\right]_{+}$mistakes to $e$ are required (then $e$ is best reply if the state consist of $\left[\frac{2}{5} N\right]_{+}$ $b$ 's, $N-\left[\frac{2}{5} N\right]_{+}-\left[\frac{23}{93} N\right]_{+} c$ 's and $\left[\frac{23}{93} N\right]_{+} e$ 's.

For $d$ to be a best reply at most $\frac{1}{10} N$ can have used strategy $b$. So, for the process to reach a state from which $d$ 's will enter, at most $\frac{1}{10} N$ can have used $b$. Now, for the best reply to change to $e$ further trembles are required, either to $b$ or to $e$. If players tremble to $b$, then at least $\left[\frac{7}{30} N\right]_{+}$mistakes are required (in this case there is positive probability of reaching a state with $\left[\frac{2}{3} N\right]_{+} b$ 's and $1-\left[\frac{2}{3} N\right]_{+} d$ 's). If players tremble to $e$, then $\left[\frac{7}{90} N\right]_{+}$ are needed (in this case there is positive probability of reaching a state with $\left[\frac{1}{10} N\right]_{-} b$ 's, $1-\left[\frac{1}{10} N\right]_{-}-\left[\frac{7}{90} N\right]_{+} d$ 's and $\left[\frac{7}{90} N\right]_{+} e^{\prime}$ s).

In conclusion, the resistance of the transition from $(N, 0,0,0,0)$ to $(0,0,0,0, N)$ is at least $\left[\frac{2}{5} N\right]_{+}+\left[\frac{7}{90} N\right]_{+}$which is larger than $\left[\frac{4}{9} N\right]_{+}$for $N$ sufficiently high. Therefore $(N, 0,0,0,0)$ is the only stochastically stable state for the KMR process. $\square$

Proposition 3.4. If $k$ is sufficiently large, then $(e, \ldots, e)$ is the unique stochastically stable state for the Young process. 
Proof: To escape from $(e, \ldots, e)$, at least $\left[\frac{4}{9} k\right]_{+}$trembles are required before the best reply changes to something different from $e$. If the trembles are all to $a$, then there is, according to the unperturbed process, positive probability of transition to $(a, \ldots, a)$. So, the resistance in the transition from $(e, \ldots, e)$ to $(a, \ldots a)$ is $\left[\frac{4}{9} k\right]_{+}$.

From $(a, \ldots, a)$, it requires $\left[\frac{2}{5} k\right]_{+}$trembles to $b$ before best reply changes to something different from $a$ (with positive probability according to the unperturbed process). Then there is positive probability according to the unperturbed process that the players in the next $\left[\frac{3}{4} k\right]_{+}$rounds all draw a sample consisting of $\left[\frac{3}{5} k\right]_{+} a$ 's and $\left[\frac{2}{5} k\right]_{-} b$ 's and consequently all choose $c$. After that, there is positive probability according to the unperturbed process that the players will sample $\left[\frac{3}{4} k\right]_{+} c^{\prime}$ 's and $\left[\frac{1}{4} k\right]_{-} a$ 's in all of the next $\left[\frac{3}{5} k\right]_{+}$rounds and consequently play $d$. Then there is positive probability according to the unperturbed process that the players sample $\left[\frac{2}{5} k\right]_{+} b$ 's and $\left[\frac{3}{5} k\right]_{-} d$ 's in all of the next $k$ rounds and hence all play $e$. Finally there is positive probability according to the unperturbed process that the players in all of the next $m-k$ rounds always sample these $k e$ 's and hence play $e$. The process has now reached the absorbing state $(e, \ldots, e)$. It follows that this is the only stochastically stable state since $\left[\frac{4}{9} k\right]_{+} \geq\left[\frac{2}{5} k\right]_{+}$for $k$ sufficiently large. $\square$

The force driving the difference in the selection this time comes from the way the players make conjectures about opponents' choice of strategy. When the players update their strategies in the KMR model, they only consider the actual play in the last round. This implies, when no trembling occurs, that a player's believed likelihood of a particular strategy that was not a best reply to his previous conjecture, cannot increase. Thus, the players' believed likelihood of a particular strategy will decrease weakly as long as this strategy is not a best reply and no trembling takes place. This is different in the Young model with incomplete sampling. Here, even without trembles, the believed likelihood of a particular strategy can increase, although this strategy was not a best reply to the conjecture held in the previous round. This follows since it is possible for two players in adjacent rounds to pick samples from different parts of the history. In the example, in order to escape "cheaply" from the equilibrium strategy $a$, first the believed likelihood of strategy $b$ has to be at least $\frac{2}{5}$, which can only happen due to trembling, but then, for the unperturbed process to continue all the way to the other absorbing state, it is necessary that the believed likelihood of $b$ first decreases and then increases. As explained above, this is possible in a Young model with incomplete sampling, but not in a KMR model. 


\section{References}

[1] Harsanyi, John.C. and Reinhard Selten (1988), A General Theory of Equilibrium in Games, Cambridge: MIT Press.

[2] Kandori, Michihiro, George J. Mailath and Rafael Rob (1993), Learning, Mutation, and Long Run Equilibria in Games, Econometrica 61, 29-56.

[3] Kandori, Michihiro, and Rafael Rob (1995), Evolution of Equilibria in the Long Run: A genreal Theory and Applications, Journal of Economic Theory 65, 383-414.

[4] Nöldeke, Georg and Larry Samuelson (1993), An Evolutionary Analysis of Backward and Forward Induction, Games and Economic Behavior 5, 425-454.

[5] Robles, Jack (1997), Evolution and Long Run Equilibria in Coordination Games with Summary of Statistic Payoff Technologies, Journal of Economic Theory 75, 180-193.

[6] Young, H. Peyton (1993), The Evolution of Conventions, Econometrica 61, 57-84. 